\title{
Microbial biogeography of the wombat gastrointestinal tract
}

\author{
Raphael Eisenhofer ${ }^{\text {Corresp., 1, } 2}$, Erin D'Agnese ${ }^{3,4}$, David Taggart ${ }^{5,6}$, Scott Carver ${ }^{7}$, Beth Penrose ${ }^{3}$ \\ ${ }^{1}$ School of Biological Sciences, University of Adelaide, Adelaide, South Australia, Australia \\ 2 Australian Research Council Centre of Excellence for Australian Biodiversity and Heritage, University of Adelaide, Adelaide, South Australia, Australia \\ 3 Tasmanian Institute of Agriculture, University of Tasmania, Hobart, Tasmania, Australia \\ 4 School of Marine and Environmental affairs, University of Washington, Seattle, Washington, United States \\ 5 School of Animal and Veterinary Sciences, University of Adelaide, Adelaide, South Australia, Australia \\ 6 FAUNA Research Alliance, Institute for Land, Water and Society, Kahibah, New South Wales, Australia \\ 7 Department of Biological Sciences, University of Tasmania, Hobart, Tasmania, Australia \\ Corresponding Author: Raphael Eisenhofer \\ Email address: raphael.eisenhoferphilipona@adelaide.edu.au
}

Most herbivorous mammals have symbiotic microbes living in their gastrointestinal tracts that help with harvesting energy from recalcitrant plant fibre. The bulk of research into these microorganisms has focused on samples collected from faeces, representing the distal region of the gastrointestinal (GI) tract. However, the Gl tract in herbivorous mammals is typically long and complex, containing different regions with distinct physicochemical properties that can structure resident microbial communities. Little work has been done to document the composition of GI microbial communities of herbivorous animals at these sites. In this study, we use 16S rRNA gene sequencing to characterize the microbial biogeography along the GI tract in two species of wombats. Specifically, we survey the microbes along four major gut regions (stomach, small intestine, proximal colon, distal colon) in a single bare-nosed wombat (Vombatus ursinus) and a single southern hairy-nosed wombat (Lasiorhinus latifrons). Our preliminary results show that GI microbial communities of wombats are structured by $\mathrm{Gl}$ region. For both wombat individuals, we observed a trend of increasing microbial diversity from stomach to distal colon. The microbial composition in the first proximal colon region was more similar between wombat species than the corresponding distal colon region in the same species. We found several microbial genera that were differentially abundant between the first proximal colon (putative site for primary plant fermentation) and distal colon regions (which resemble faecal samples). Surprisingly, only $10.6 \%$ (99) and $18.7 \%$ (204) of amplicon sequence variants (ASVs) were shared between the first proximal colon region and the distal colon region for the bare-nosed and southern hairy-nosed wombat, respectively. These results suggest that microbial communities in the first proximal colon region-the putative site of primary plant fermentation in wombats-are distinct from the 
distal colon, and that faecal samples may have limitations in capturing the diversity of these communities. While faeces are still a valuable and effective means of characterising the distal colon microbiota, future work seeking to better understand how GI microbiota impact the energy economy of wombats (and potentially other hindgut-fermenting mammals) may need to take gut biogeography into account. 
1 Microbial biogeography of the wombat gastrointestinal tract

\section{Authors}

4 Raphael Eisenhofer ${ }^{1,2}$, Erin D’Agnese ${ }^{3,4}$, David Taggart ${ }^{5,6}$, Scott $^{\prime}$ Carver $^{7}$ and Beth Penrose ${ }^{3}$

5

1. School of Biological Sciences, University of Adelaide, Adelaide, South Australia, Australia

2. Australian Research Council Centre for Australian Biodiversity and Heritage, University of Adelaide, Adelaide, South Australia, Australia

3. Tasmanian Institute of Agriculture, University of Tasmania, Hobart, Tasmania, Australia

4. School of Marine and Environmental affairs, University of Washington, Seattle, Washington, USA

5. School of Animal and Veterinary Sciences (Waite), University of Adelaide, Adelaide, South Australia, Australia

6. FAUNA Research Alliance, PO Box 5092, Kahibah, NSW, 2290, Australia

7. Department of Biological Sciences, University of Tasmania, Hobart, Tasmania, Australia

Corresponding Author:

Raphael Eisenhofer ${ }^{1,2}$

North Terrace Campus, Adelaide, South Australia, 5005, Australia

Email address: raph.eisenhofer@gmail.com

\section{Abstract}

Most herbivorous mammals have symbiotic microbes living in their gastrointestinal tracts that help with harvesting energy from recalcitrant plant fibre. The bulk of research into these microorganisms has focused on samples collected from faeces, representing the distal region of the gastrointestinal $(\mathrm{Gl})$ tract. However, the $\mathrm{Gl}$ tract in herbivorous mammals is typically long 


\section{7}

and complex, containing different regions with distinct physico-chemical properties that can structure resident microbial communities. Little work has been done to document the composition of GI microbial communities of herbivorous animals at these sites. In this study, we use $16 \mathrm{~S}$ rRNA gene sequencing to characterize the microbial biogeography along the $\mathrm{Gl}$ tract in two species of wombats. Specifically, we survey the microbes along four major gut regions (stomach, small intestine, proximal colon, distal colon) in a single bare-nosed wombat (Vombatus ursinus) and a single southern hairy-nosed wombat (Lasiorhinus latifrons). Our preliminary results show that GI microbial communities of wombats are structured by GI region. For both wombat individuals, we observed a trend of increasing microbial diversity from stomach to distal colon. The microbial composition in the first proximal colon region was more similar between wombat species than the corresponding distal colon region in the same species. We found several microbial genera that were differentially abundant between the first proximal colon (putative site for primary plant fermentation) and distal colon regions (which resemble faecal samples). Surprisingly, only $10.6 \%$ (99) and $18.7 \%$ (204) of amplicon sequence variants (ASVs) were shared between the first proximal colon region and the distal colon region for the bare-nosed and southern hairy-nosed wombat, respectively.

These results suggest that microbial communities in the first proximal colon region-the putative site of primary plant fermentation in wombats-are distinct from the distal colon, and that faecal samples may have limitations in capturing the diversity of these communities. While faeces are still a valuable and effective means of characterising the distal colon microbiota, future work seeking to better understand how GI microbiota impact the energy economy of wombats (and potentially other hindgut-fermenting mammals) may need to take gut biogeography into account. 
51 gastrointestinal tract. These microbes are instrumental in digesting food, producing nutrients for

52 the host, and as a potential first barrier to invading pathogens (reviewed in (Rowland et al. 2018;

53 Ubeda, Djukovic, and Isaac 2017)). In herbivores specifically, gut microbes break down cellulose

54 and other plant cellular components so they may be used as nutrients by the animal (Flint et al.

55 2012), and can detoxify plant defense compounds (Dearing and Kohl 2017). Without these

56 microbes there would be little to no nutritional value in much of the plant matter herbivorous

57 mammals are known to consume and thrive on. In ruminants, the bulk of microbial digestion and

58 fermentation occurs in a highly modified foregut (rumen) where nutrients are broken down by

59 rumen microbiota and made ready for absorption through the small intestine (Immig 1996).

60 However, in herbivores without a rumen, the microbial breakdown of plant matter into usable

61 nutrients and energy occurs in the latter parts of the gut-principally the cecum and colon

62 (Stevens and Hume 1998). Prior research has found that microbial communities vary along the

63 mammalian GI tract (Donaldson, Lee, and Mazmanian 2015; Costa et al. 2015; Ericsson et al.

64 2016; Kelly et al. 2017; Crespo-Piazuelo et al. 2018; Eckburg et al. 2005; Flynn et al. 2018;

65 Vasapolli et al. 2019). In mice, large differences were observed between cecum, distal colon, and

66 faeces microbial communities (Gu et al. 2013; Pang et al. 2012). Marked microbial community

67 differences between caecal, colon, and faecal samples were also identified in a study on koalas,

68 which are in the same family as wombats (Barker et al. 2013). How representative faecal samples

69 are to other parts of the GI tract is likely host species specific, resulting from variations in GI

70 morphology and function. Yet our understanding of these differences outside of domesticated

71 placental mammals remains unstudied. As a major goal of gut microbiome research is to

72 understand how microbes influence host health and ecology, efforts should be made to better

73 characterize the site-specific nature of microbes along the $\mathrm{Gl}$ tract and determine how

74 representative faecal samples are. 
Wombats represent a useful herbivorous hindgut fermenting group to contribute to

76 knowledge on microbial community differences along the Gl tract. As marsupials, they are a 77 valuable taxonomic group to add to a growing body of GI tract microbiota research. Bare-nosed 78 (Vombatus ursinus, hereafter BNW) and Southern hairy-nosed (Lasiorhinus latifrons, hereafter 79 SHNW) wombats represent two of the three extant wombat species and are also in the same sub80 order as the koala (Diprotodontia). The BNW and SHNWs are large (20-35 kg), fossorial, 81 metabolically depressed, nocturnal, and largely solitary marsupials found allopatrically across 82 large areas of southeastern and southern Australia (Wells 1989). These two wombat species also 83 represent temperate and arid adapted grazing diets, respectively (Wells 1989; Camp et al. 2020), 84 and are thought to have shared a common ancestor $\sim 8$ million years ago (Mitchell et al. 2014). 85 The SHNW has a comparatively longer distal colon, with the BNW possessing a wider digestive tract and greater proximal colon surface area (Figure 1) (Barboza and Hume 1992a; Hume 1999).

87 Both species have caeca that are smaller and less functional than other hindgut fermenters, and the digesta passage rates are thought to be the same between wombat species (Hume 1999). Previous research measuring short chain fatty acids indicates that most plant fermentation occurs in the proximal colon of both wombat species rather than in other regions of the GI tract (Barboza and Hume 1992b). It has been estimated that plant fermentation accounts for $>60 \%$ of a wombat's daily energy intake (Barboza and Hume 1992b). To date, gut microbiota research in wombats has focused on faecal samples obtained from captive (Shiffman et al. 2017) and wild wombats (Eisenhofer, Helgen, and Taggart 2021; Weiss et al. 2021), with nothing known about how microbial communities are structured throughout the $\mathrm{Gl}$ tract, and how representative faeces are to other GI regions. changes along the GI tract of two wombat individuals, a wild BNW and SHNW. We predicted that the proximal colon, the putative primary site of plant fermentation in wombats, would harbor a 
100 distinct microbial community to that of the rest of the gastrointestinal tract, including the distal

101 colon in these wombat species.

102

\section{Materials \& Methods}

104 Sample collection

105 Sample collection occurred during necropsies on two deceased wild wombats $(n=2)$, one BNW 106 and one SHNW. A $17.1 \mathrm{~kg}$ female BNW was hit by a vehicle in the New Norfolk area, Tasmania, 107 Australia and taken to Bonorong Wildlife Sanctuary. Injuries to this wombat were too severe for 108 rehabilitation, and it was euthanized on 13th November 2019. Immediately following euthanasia 109 the carcass was placed in a $-20^{\circ} \mathrm{C}$ freezer at the University of Tasmania, Hobart. The SHNW was 110 captured near Swan Reach in the Murraylands of South Australia, approximately 100 km north111 east of Adelaide, as part of an ecological study on seasonal reproduction and breeding, but then

112 died later of an upper respiratory tract infection. The carcass was driven to Adelaide and put into $113 \mathrm{a}-20^{\circ} \mathrm{C}$ freezer within 2 hours of death. Given that storage of faecal samples for extended periods 114 at room-temperature can result in microbial 'blooming' and distorted taxonomic compositions, the 115 rapid freezing of the samples in this study (both within 8 hours of death) should help mitigate such 116 biases (Song et al. 2016; Lauber et al. 2010). Regardless, any biological differences between 117 wombat species should outweigh any technical biases introduced during sample storage (Song 118 et al. 2016; Lauber et al. 2010).

119 Prior to necropsy, carcasses were put into a $4^{\circ} \mathrm{C}$ refrigerator and defrosted for $\sim 48 \mathrm{~h}$. In human 120 faecal samples, freeze-thawing was only found to influence taxon abundance after the fourth cycle 121 of freeze-thaw (Gorzelak et al. 2015). The entire digestive systems were then removed from the 122 abdominal cavity and the functional sections of the GI tract were labeled as per (Barboza and 123 Hume 1992a). For the stomach, small intestine, and proximal colon, samples were collected in 124 the middle of each functional section (Figure 1). For distal colon samples, the BNW sample was 
125 collected halfway through the distal colon. For the SHNW four samples were collected: DC1 was 126 collected 1 meter down from the proximal colon/distal colon boundary, DC2 was collected 2 127 meters down, etc. Samples of the digesta were collected in duplicate in the SHNW and in triplicate 128 in the BNW. While faecal samples could not be collected from the euthanized animals, the distal 129 colon samples collected resembled faeces, being similar in size, shape, and moisture content. To 130 avoid cross-contamination between sites the digesta was squeezed directly into individual sterile 131 containers. Samples were then stored at $-20^{\circ} \mathrm{C}$ prior to molecular work.

DNA extraction and 16S rRNA gene library preparation

134 DNA extraction was performed using the QIAamp® Fast DNA stool mini kit from QIAGEN 135 according to the manufacturer's protocol (Qiagen Pty. limited Victoria, Australia). Duplicate samples from each GI region for both wombat species were processed. BNW samples were extracted at the University of Tasmania, and SHNW samples were extracted at the University of Adelaide. BNW DNA extracts were then shipped frozen to the University of Adelaide for library preparation. Barcoded V4 region 16S rRNA gene amplicons were generated using primers from (Caporaso et al. 2011) (forward primer 515F: GTGCCAGCMGCCGCGGTAA and barcoded 806R: GGACTACHVGGGTWTCTAAT). The PCR reactions were prepared in a pre-PCR laboratory in a 5\% sodium hypochlorite-cleaned and UV irradiated hood. Single reactions (Marotz et al. 2019) of $2.5 \mu \mathrm{L}$ X10 HiFi buffer, $0.1 \mu \mathrm{L}$ Platinum ${ }^{\mathrm{TM}}$ Taq DNA Polymerase (ThermoFisher), 19.2 $\mu \mathrm{L} \mathrm{dH} 2 \mathrm{O}, 0.2 \mu \mathrm{L} 100 \mathrm{mM}$ dNTP mix, $0.5 \mu \mathrm{L}$ each of $10 \mu \mathrm{M}$ forward and reverse primer and $1 \mu \mathrm{L}$ input DNA. The DNA was amplified using an initial denaturation at $94{ }^{\circ} \mathrm{C}$ for $3 \mathrm{~min}$, followed

147 by 35 cycles of denaturation at $94{ }^{\circ} \mathrm{C}$ for $45 \mathrm{~s}$, annealing at $50{ }^{\circ} \mathrm{C}$ for $1 \mathrm{~min}$, elongation at $68^{\circ} \mathrm{C}$ 148 for $90 \mathrm{~s}$, with final adenylation for $10 \mathrm{~min}$ at $68{ }^{\circ} \mathrm{C}$ (Thompson et al. 2017). Gel electrophoresis 149 was performed on PCR reactions on a 3.5\% agarose gel to ensure samples contained amplicons 150 of the desired length ( $390 \mathrm{bp}$ ). For each sample, $1 \mu \mathrm{L}$ PCR amplified DNA was mixed into $199 \mu \mathrm{L}$ 
151 Qubit $\circledast$ working solution (diluted Qubit $®$ dsDNA HS Reagent 1:200 in Qubit $®$ dsDNA HS Buffer)

152 and quantified using a Qubit® 2.0 Fluorometer. Samples were then pooled equimolar and cleaned

153 using AxyPrep ${ }^{\mathrm{TM}}$ (Axygen) following the manufacturer's instructions. The final pool was quantified

154 and quality checked using an Agilent TapeStation. DNA sequencing was performed on an Illumina

155 MiSeq (v2, $2 \times 150 \mathrm{bp})$ at SAHMRI (South Australian Health and Medical Research Institute).

156

157 Bioinformatic analyses

158 QIIME2 (Bolyen et al. 2019) and R v4.0.2 (R Core Team 2020) were used to perform the

159 bioinformatic analyses and create figures. Reproducible code is publicly available at the following

160 GitHub repository (https://github.com/EisenRa/2021 Wombat Gl tract 16S). Forward reads

161 were denoised using deblur (Amir et al. 2017) in QIIME2. Representative sequences were

162 assigned taxonomy using the QIIME2 feature-classifier plugin (Bokulich et al. 2018) on the pre-

163 trained SILVA (Quast et al. 2013) 138 V4 region classifier. A phylogenetic tree was created using

164 the SATé-enabled phylogenetic placement (SEPP) technique (Janssen et al. 2018) using the

165 fragment-insertion QIIME2 plugin. Data was then imported into rStudio into a phyloseq (McMurdie

166 and Holmes 2013) object using qiime2R (https://github.com/jbisanz/qiime2R), and manipulated

167 with dplyr (https://github.com/tidyverse/dplyr), tidyr (https://github.com/tidyverse/tidyr/), and the

168 microbiome package (https://github.com/microbiome/microbiome/). For all sample type

169 comparisons, the table was rarefied at a depth of 21,710 , for colon-only comparisons the table

170 was rarefied to 47,301 . ASVs that were found in only 1 single replicate of a gut region were

171 removed. These ASVs represented only $4.3 \%$ of identified ASVs and $0.16 \%$ of the mean relative

172 abundance of the dataset. Alpha and beta diversity measures (including UniFrac (Lozupone and

173 Knight 2005)) were calculated in R using phyloseq, and figures were plotted using ggplot2

174 (Wickham 2016) and ggVennDiagram (Gao, Yu, and Cai 2021). Compositional analysis of the

175 community was done using ANCOM-II in R (ANCOM v2.1 (Kaul et al. 2017) in R v.4.0.3). Analysis

176 was undertaken using an ANCOM - adjusted for covariates model which accounts for the 
177 covariation expected at the species/animal level. The analysis was performed at the rarefied

178 genus level for the proximal colon and distal colon samples only. For the venn and euler diagrams, 179 ASVs needed more than 2 reads assigned at a given site (between two replicates) to be 180 considered detected, and were then plotted using ggVenDiagram 181 (https://github.com/gaospecial/ggVennDiagram).

182

183 Results

184 DNA sequencing of the 30 gastrointestinal digesta samples (Figure 1) and 1 extraction blank 185 control $(E B C)$ resulted in 4,923,858 (mean of 158,834 $\pm 76,389$ ) forward reads (R1), which were 186 denoised using deblur into 2,524 amplicon sequence variants (ASVs).

187

188 Microbial biogeography through the digestive tract of two wombat species

189 To test if these different gut regions influenced the microbial communities, we analyzed the 190 microbial composition, richness (the number of ASVs), and diversity of duplicate luminal samples 191 taken throughout the $\mathrm{Gl}$ tract of each wombat species. We first investigated what types of microbes (the taxonomic composition) were present in the different GI samples for both wombat species. At the phylum level, stomach and small intestine samples tended to be dominated by Proteobacteria, Firmicutes, and Fusobacteriota (SI Figure 1) We observed a large difference in phyla composition between the proximal (PSI) and distal small intestine (DSI) samples from the SHNW, with the PSI dominated by Proteobacteria, and DSI dominated by Fusobacteria (SI Figure

1). The dominant families in the stomach and small intestine samples were Pasteurellaceae (59\% in BNW-ST (ST = Stomach), 56\% in SHNW-ST, 43\% in SHNW-PSI), Peptostreptococcaceae 199 (67\% in BNW-SI), and Fusobacteriaceae (81\% in SHNW-DSI) (Figure 2). In contrast, the colon 200 samples for both wombat species were dominated by the Bacteroidota, Firmicutes, and 201 Spirochaetota phyla (SI Figure 1). We also observed taxonomic differences between proximal 
202 (especially proximal colon 1\&2) and distal colon samples for both the wombat species (Figure 2).

203 The first proximal colon samples were dominated by Prevotellaceae ( $40 \%$ relative abundance), 204 and Fibrobacteraceae ( $10 \%$ for the BNW), whereas the latter proximal colon and distal colon 205 samples contained higher proportions of Spirochaetaceae, Rikenellaceae, and WCHB1-41. A 206 heatmap of all microbial families is available in SI Figure 4.

207

208 Microbial richness tended to increase through the length of the digestive tract for both wombat 209 species (Figure 3). Stomach and small intestine samples had the lowest ASV richness ( 100210 200), followed by the first two proximal colon samples (PC1/PC2: 400-500), with the latter 211 proximal colon and distal colon samples exhibiting the highest ASV richness ( 500-800). Due to 212 our limited sampling, we were unable to run linear models to confirm this pattern statistically, and 213 future studies employing larger sample sizes should test the observed pattern.

215 Like richness, the types of microorganisms living in a site and how they are structured 216 (composition) can also be influenced by the environmental factors of that site. To test this, we 217 compared the microbial community structure of these distinct sites using both the abundance 218 weighted and unweighted UniFrac distance metrics. As predicted, major differences were 219 observed in microbial composition along the Gl tract in both wombat individuals, with stomach, 220 small intestine colon, and colon samples clustering across the axis of most variation (Axis 1: 221 Figure 4A/B). Note that the colon samples are likely driving the placement of samples on the first 222 two axes. Colon samples from the different wombats were separated across axis 2 for the 223 unweighted UniFrac analysis (Figure 4A), and separation between proximal and distal colon 224 samples was observed for both unweighted and weighted UniFrac distances (Figure 4). 225 Interestingly, the microbial composition of the first proximal colon samples of different wombat 226 species is more similar to each other than they are to the distal colon samples of the same species 227 (Figure 4B, SI Figure 2B). Considering colon samples only, potentially species-specific 
228 differences in abundance-weighted microbial composition between distal colon samples were 229 observed across axis 2 (SI Figure 2B). Note however, that we only have 1 sample per species, 230 and can therefore not account for intra-species diversity. These results indicate that microbial 231 diversity and composition vary throughout the GI tract of two hindgut fermenting species, and that 232 the start of the proximal colon--the putative primary site of fermentation in these wombat species 233 -- is distinct from the distal colon.

234

\section{Microbial differences between proximal and distal colon sites}

236 Because the proximal colon is the putative primary site of plant fermentation in wombats, we next 237 focused on further characterising microbial community compositional differences between the 238 proximal and distal colons for both species. As there were many ASVs that were classified to the same taxa we collapsed the ASV table to the genus-level, and ran ANCOM-II to identify genera that were differentially abundant between different regions of the colon (i.e. PC1, PC2, DC, etc.).

Of the 253 genera classified in the dataset, ANCOM-II identified 60 that were significantly differentially abundant throughout the colon when the W-statistic threshold for rejecting the null

was at $70 \%$ (Figure 5) (SI Table 1). Taxa that had significantly higher abundance in PC1/2 vs. DC include: Prevotella (W=245), Prevotellaceae_UCG-001 (W=241), Bacteroides $(\mathrm{W}=239)$, and Bacteroidales_RF16_group (W=231). Taxa with a higher relative abundance in DC vs. PC1/2 include: Bacteroidales_BS11_gut_group (W=250), Bacteroidales_UCG-001 (W=243), WCHB1$41(\mathrm{~W}=242)$, and Izemoplasmatales $(\mathrm{W}=226)$.

To test how representative the microbial community of faeces are to the first proximal colon site, we measured how many ASVs were shared between PC1 and the last DC sample for each wombat species. Surprisingly, only 99 (10.6\%) and 204 (18.8\%) ASVs were shared between PC1

252 and DC samples for the BNW and SHNW, respectively (Figure 6). See (SI Figure 3) for a cross253 species comparison. The ASVs that were unique to the BNW PC1 and DC sites accounted for 
$25425 \%$ and $64 \%$ of the relative abundance in those sites, respectively. Likewise, the ASVs that were 255 unique to the SHNW PC1 and DC sites accounted for $32 \%$ and $67 \%$, respectively. These 256 differences remained after collapsing ASVs to the genus-level (SI Figure 5), albeit with a smaller 257 magnitude (relative abundance of site-specific genera: BNW DC $=5 \%$ BNW PC $=20 \%$ SHNW $258 \mathrm{DC}=3 \%$ SHNW PC $=12 \%)$. These results indicate that the first proximal colon and distal colon 259 sites harbour distinct communities of microbes.

260

\section{Discussion}

262 Mammalian gut microbial communities play vital roles in the harvesting of energy and nutrients 263 (Rowland et al. 2018). Due to ease of sampling, most previous mammalian gut microbiota 264 research has focused on faecal samples as proxies for the gut microbiota. However, the 265 mammalian GI tract contains a series of distinct microbial growth conditions that can structure the 266 diversity and composition of resident microbial communities (Donaldson, Lee, and Mazmanian 267 2015). Outside of model or domesticated placental mammal species there has been little work in 268 characterising how GI microbial communities are structured along GI tracts, and how 269 representative faeces are of other GI regions. Our work here fills this gap for two species of free270 living, large, hindgut-fermenting marsupials—the bare-nosed wombat (BNW) and the southern 271 hairy-nosed wombat (SHNW). Although we had data for only two individual wombats, our results 272 showed that microbial community composition, structure and diversity varied along the wombat 273 GI tract, and that the first proximal colon region—the putative primary site of fermentation-was 274 highly dissimilar to the distal colon. Our findings also indicate that faecal samples collected for 275 wombats may not be representative of the primary site of fermentation. Future studies seeking to 276 understand the roles that GI microbial communities play in host energy acquisition and health 277 should consider the microbial biogeography of the mammalian GI tract. 278 
279 A major finding was that the microbial communities present in the first proximal colon region (PC1)

280 were more similar between both individuals, than to subsequent colon region samples from the

281 same individual. This is particularly interesting given that both individuals are from different

282 species - though still preliminary given the small sample size in our study ( $\mathrm{n}=1$ for both species).

283 PC1 in both wombat individuals were dominated by the family Prevotellaceae ( $40 \%$ relative 284 abundance), a family that contains members with extensive carbohydrate/fiber fermentation 285 capabilities (Filippo et al. 2010; Kovatcheva-Datchary et al. 2015). All other GI sites contained 286 lower levels of Prevotellaceae ( $<5 \%$ relative abundance). In addition, PC1 for both wombat 287 individuals contained significantly higher levels of bacteria classified to the genus Bacteroides, 288 which are known to possess a diverse suite of carbohydrate-active enzymes (Kaoutari et al. 289 2013). PC1 of the BNW had relatively high levels $(\sim 10 \%$ relative abundance) of the genus 290 Fibrobacter ( 0.3\% in the SHNW PC1), which have known fiber-degrading capacity and have 291 been found in various placental foregut- and hindgut-fermenting herbivores (Neumann, 292 McCormick, and Suen 2017). These distinct microbial compositions in PC1 of both wombat 293 individuals compared to subsequent colon regions warrant further investigation with shotgun 294 metagenomics to verify whether these taxonomic differences represent differences in the capacity to ferment fibre. This would further test Barboza and Hume's finding that PC1 represents the primary site of microbial fermentation in the wombat GI tract, and offer us a greater understanding of microbial fermentation in wombats. Finally, such a study could also verify whether the high proportion of Firmicutes found in colon samples are involved fibre degradation as expected 299 (Biddle et al. 2013).

300

301 Given the major microbial compositional differences observed between proximal and distal colon 302 samples, we sought to determine how many proximal colon-associated microbes would be 303 detected by sampling faeces, the most commonly used sample type in mammalian gut microbiota 304 research. Surprisingly, only a very small proportion of PC1 ASVs could be detected in distal colon 
305

306

307

308

309

310

311

312

313

314

315

316

317

318

319

320

321

322

323

324

325

326

327

328

329

330

samples $(10.6 \%$ and $18.7 \%$ in the BNW and SHNW, respectively). This suggests that seeking to understand microbial functions at the putative primary site of fermentation through faecal samples may not be feasible. This is not to say that faecal samples are not useful, as previous gut microbiome research on captive (Shiffman et al. 2017) and wild wombats (Eisenhofer, Helgen, and Taggart 2021; Weiss et al. 2021) have yielded insights into wombat digestion and ecology. Eisenhofer et al. used faecal samples from both captive and wild SHNWs to show that captivity has a large influence on the faecal microbiota of SHNWs. They were also able to identify population-specific microbial signatures and found a correlation between habitat type (degraded vs. intact) and faecal microbiota composition in SHNWs (Eisenhofer, Helgen, and Taggart 2021). Shiffman et al. used shotgun metagenomic sequencing on faecal samples collected from a captive SHNW, and found numerous microbial genes involved in plant degradation and urea recycling (Shiffman et al. 2017). Interestingly, they made note that they could not detect bacteria from the phylum Fibrobacterota, which are commonly found in herbivorous mammals (Neumann, McCormick, and Suen 2017). We identified Fibrobacterota in both wombat species, with a higher relative abundance in the BNW, particularly in the proximal colon. Overall, our results suggest that faeces may not be a representative sample type for the putative primary site of microbial fermentation in wombats. We propose that future research seeking to understand the roles that GI microbes play in wombat (or other mammal) digestion consider this, and aim to study other regions of the $\mathrm{GI}$ tract where possible.

Our study is not without limitations. The opportunistic nature of sample collection from fresh necropsied animals limited this study to only one animal for each wombat species. This hampered our ability to compare microbial differences between wombat species, as the differences observed may be related to individual-level variation-making us unable to run statistical tests on GI tract alpha or beta diversity. However, the consistency observed between the biological replicates and in overall trends for both species of wombat support our findings relating to PC1/distal microbial 
331 differences and the level of representability of the faeces to PC1—despite the ecological

332 differences between wombat species and the estimated $\sim 8$ million years since they last shared a 333 common ancestor (Mitchell et al. 2014). Another limitation is that we could not distinguish DNA

334 from living or dead cells, and it is possible that the differences between GI sites is greater than 335 measured in our study due to dead cells/relic DNA moving from site to site and inflating similarity 336 (Lennon et al. 2018). Future studies with larger sample sizes will enable more detailed microbial 337 comparisons between wombat species, and improve estimates of within-animal microbial 338 differences along the GI tract.

339

340 The findings of our study have spawned several promising avenues of future research. Using 341 shotgun metagenomics to compare and contrast the microbial functions present between 342 proximal and distal colons could allow for a greater understanding of the roles GI microbes play 343 in wombat digestion and health. The strain-level information obtained from shotgun 344 metagenomics would also allow for greater tracking of microbes along the GI tract, and help 345 disentangle issues relating to the over splitting of microbes due to intragenomic heterogeneity in 346 16S rRNA genes (Sun et al. 2013). Future, larger sample size comparisons between wombat 347 species could also seek to identify microbes that are shared between wombat species, and 348 consistently found within species. Any such 'core' microbes that are still shared between wombat 349 species despite $\sim 8$ million years of separation could indicate that they are important for host 350 health. In our study the distal colon exhibited the greatest microbial differentiation between 351 wombat species. Such interspecies microbial differences in the latter parts of the colon could be 352 due to GI morphological/physiological differences between wombat species (Barboza and Hume 353 1992a) and relate to differences in the ecology of the two wombat species. Further larger-scale 354 comparisons between BNW (mesic living) and SHNWs (arid/semi-arid living), and other 355 phylogenetically distinct mammals could offer insights into the potential roles that GI microbes 356 play in the arid and temperate adaptability of mammals in Australia. 


\section{Conclusions}

359 Our study suggests that microbial communities in the first proximal colon region —-the putative site 360 of primary plant fermentation in wombats-are distinct from the distal colon, and that faecal 361 samples may have limitations in capturing the diversity of these communities. While faeces are 362 still a valuable and effective means of characterising the distal colon microbiota, future work 363 seeking to better understand how GI microbes impact the energy economy of wombats (and 364 potentially other hindgut-fermenting mammals) may need to take gut biogeography into account.

\section{Abbreviations}

366 ANCOM: Analysis of Composition of Microbiomes

367 ASV: Amplicon Sequence Variant

368 BNW: Bare-Nosed Wombat

369 DC: Distal Colon

370 DSI: Distal Small Intestine

371 GI: Gastrointestinal

372 PC: Proximal Colon

373 PCoA: Principal Coordinates Analysis

374 PCR: Polymerase Chain Reaction

375 PSI: Proximal Small Intestine

376 SI: Small Intestine

377 SHNW: Southern Hairy-Nosed Wombat

378 ST: Stomach 


\section{References}

380

381

382

383

384

385

386

387

388

389

390

391

392

393

394

395

396

397

398

399

400

401

402

403

404

405

406

407

408

409

410

411

412

413

414

415

416

417

418

419

420

421

422

423
Amir, Amnon, Daniel McDonald, Jose A. Navas-Molina, Evguenia Kopylova, James T. Morton, Zhenjiang Zech Xu, Eric P. Kightley, et al. 2017. 'Deblur Rapidly Resolves Single-Nucleotide Community Sequence Patterns'. MSystems 2 (2): e00191-16. https://doi.org/10.1128/mSystems.00191-16.

Barboza, P. S., and I. D. Hume. 1992a. 'Digestive Tract Morphology and Digestion in the Wombats (Marsupialia: Vombatidae)'. Journal of Comparative Physiology $B$ 162 (6): 552-60. https://doi.org/10.1007/BF00264817.

Barboza, P. S., and I. D. Hume. 1992b. 'Hindgut Fermentation in the Wombats: Two Marsupial Grazers'. Journal of Comparative Physiology B 162 (6): 561-66. https://doi.org/10.1007/BF00264818.

Barker, Christopher J., Amber Gillett, Adam Polkinghorne, and Peter Timms. 2013. 'Investigation of the Koala (Phascolarctos Cinereus) Hindgut Microbiome via $16 \mathrm{~S}$ Pyrosequencing'. Veterinary Microbiology 167 (3): 554-64. https://doi.org/10.1016/j.vetmic.2013.08.025.

Biddle, Amy, Lucy Stewart, Jeffrey Blanchard, and Susan Leschine. 2013. 'Untangling the Genetic Basis of Fibrolytic Specialization by Lachnospiraceae and Ruminococcaceae in Diverse Gut Communities'. Diversity 5 (3): 627-40. https://doi.org/10.3390/d5030627.

Bokulich, Nicholas A., Benjamin D. Kaehler, Jai Ram Rideout, Matthew Dillon, Evan Bolyen, Rob Knight, Gavin A. Huttley, and J. Gregory Caporaso. 2018. 'Optimizing Taxonomic Classification of Marker-Gene Amplicon Sequences with QIIME 2's Q2-Feature-Classifier Plugin'. Microbiome 6 (May): 90. https://doi.org/10.1186/s40168-018-0470-z.

Bolyen, Evan, Jai Ram Rideout, Matthew R. Dillon, Nicholas A. Bokulich, Christian C. Abnet, Gabriel A. Al-Ghalith, Harriet Alexander, Eric J. Alm, Manimozhiyan Arumugam, and Francesco Asnicar. 2019. 'Reproducible, Interactive, Scalable and Extensible Microbiome Data Science Using QIIME 2'. Nature Biotechnology 37 (8): 852-57.

Camp, Amanda, Adam E. Croxford, Caroline S. Ford, Ute Baumann, Peter R. Clements, Stefan Hiendleder, Lucy Woolford, et al. 2020. 'Dual-Locus DNA Metabarcoding Reveals Southern Hairy-Nosed Wombats (Lasiorhinus Latifrons Owen) Have a Summer Diet Dominated by Toxic Invasive Plants'. PLOS ONE 15 (3): e0229390. https://doi.org/10.1371/journal.pone.0229390.

Caporaso, J. Gregory, Christian L. Lauber, William A. Walters, Donna Berg-Lyons, Catherine A. Lozupone, Peter J. Turnbaugh, Noah Fierer, and Rob Knight. 2011. 'Global Patterns of 16S RRNA Diversity at a Depth of Millions of Sequences per Sample'. Proceedings of the National Academy of Sciences 108 (Supplement 1): 4516-22. https://doi.org/10.1073/pnas.1000080107.

Costa, M. C., G. Silva, R. V. Ramos, H. R. Staempfli, L. G. Arroyo, P. Kim, and J. S. Weese. 2015. 'Characterization and Comparison of the Bacterial Microbiota in Different Gastrointestinal Tract Compartments in Horses'. The Veterinary Journal 205 (1): 74-80. https://doi.org/10.1016/j.tvjl.2015.03.018.

Crespo-Piazuelo, Daniel, Jordi Estellé, Manuel Revilla, Lourdes Criado-Mesas, Yuliaxis Ramayo-Caldas, Cristina Óvilo, Ana I. Fernández, Maria Ballester, and Josep M. 
424

425

426

427

428

429

430

431

432

433

434

435

436

437

438

439

440

441

442

443

444

445

446

447

448

449

450

451

452

453

454

455

456

457

458

459

460

461

462

463

464

465

466

467

468
Folch. 2018. 'Characterization of Bacterial Microbiota Compositions along the Intestinal Tract in Pigs and Their Interactions and Functions'. Scientific Reports 8 (1): 12727. https://doi.org/10.1038/s41598-018-30932-6.

Dearing, M. Denise, and Kevin D. Kohl. 2017. 'Beyond Fermentation: Other Important Services Provided to Endothermic Herbivores by Their Gut Microbiota'. Integrative and Comparative Biology 57 (4): 723-31. https://doi.org/10.1093/icb/icx020.

Donaldson, Gregory P., S. Melanie Lee, and Sarkis K. Mazmanian. 2015. 'Gut Biogeography of the Bacterial Microbiota'. Nature Reviews Microbiology advance online publication (October). https://doi.org/10.1038/nrmicro3552.

Eckburg, Paul B., Elisabeth M. Bik, Charles N. Bernstein, Elizabeth Purdom, Les Dethlefsen, Michael Sargent, Steven R. Gill, Karen E. Nelson, and David A. Relman. 2005. 'Diversity of the Human Intestinal Microbial Flora'. Science 308 (5728): 1635-38. https://doi.org/10.1126/science.1110591.

Eisenhofer, Raphael, Kristofer M. Helgen, and David Taggart. 2021. 'Signatures of Landscape and Captivity in the Gut Microbiota of Southern Hairy-Nosed Wombats (Lasiorhinus Latifrons)'. Animal Microbiome 3 (1): 4. https://doi.org/10.1186/s42523-020-00068-y.

Ericsson, Aaron C., Philip J. Johnson, Marco A. Lopes, Sonja C. Perry, and Hannah R. Lanter. 2016. 'A Microbiological Map of the Healthy Equine Gastrointestinal Tract'. PLOS ONE 11 (11): e0166523. https://doi.org/10.1371/journal.pone.0166523.

Filippo, Carlotta De, Duccio Cavalieri, Monica Di Paola, Matteo Ramazzotti, Jean Baptiste Poullet, Sebastien Massart, Silvia Collini, Giuseppe Pieraccini, and Paolo Lionetti. 2010. 'Impact of Diet in Shaping Gut Microbiota Revealed by a Comparative Study in Children from Europe and Rural Africa'. Proceedings of the National Academy of Sciences 107 (33): 14691-96. https://doi.org/10.1073/pnas.1005963107.

Flint, Harry J., Karen P. Scott, Sylvia H. Duncan, Petra Louis, and Evelyne Forano. 2012. 'Microbial Degradation of Complex Carbohydrates in the Gut'. Gut Microbes 3 (4): 289-306. https://doi.org/10.4161/gmic.19897.

Flynn, Kaitlin J., Mack T. Ruffin, D. Kim Turgeon, and Patrick D. Schloss. 2018. 'Spatial Variation of the Native Colon Microbiota in Healthy Adults'. Cancer Prevention Research 11 (7): 393-402. https://doi.org/10.1158/1940-6207.CAPR-17-0370.

Gao, Chun-Hui, Guangchuang Yu, and Peng Cai. 2021. 'GgVennDiagram: An Intuitive, Easy-to-Use, and Highly Customizable R Package to Generate Venn Diagram'. Frontiers in Genetics 12. https://www.frontiersin.org/article/10.3389/fgene.2021.706907.

Gorzelak, Monika A., Sandeep K. Gill, Nishat Tasnim, Zahra Ahmadi-Vand, Michael Jay, and Deanna L. Gibson. 2015. 'Methods for Improving Human Gut Microbiome Data by Reducing Variability through Sample Processing and Storage of Stool'. PloS One 10 (8): e0134802. https://doi.org/10.1371/journal.pone.0134802.

Gu, Shenghua, Dandan Chen, Jin-Na Zhang, Xiaoman Lv, Kun Wang, Li-Ping Duan, Yong Nie, and Xiao-Lei Wu. 2013. 'Bacterial Community Mapping of the Mouse

Peer] reviewing PDF | (2021:09:65718:2:0:NEW 25 Jan 2022) 
469

470

471

472

473

474

475

476

477

478

479

480

481

482

483

484

485

486

487

488

489

490

491

492

493

494

495

496

497

498

499

500

501

502

503

504

505

506

507

508

509

510

511

512

513

514

Gastrointestinal Tract'. PLOS ONE 8 (10): e74957.

https://doi.org/10.1371/journal.pone.0074957.

Hume, lan D. 1999. Marsupial Nutrition. New York: Cambridge University Press.

Immig, Irmgard. 1996. 'The Rumen and Hindgut as Source of Ruminant

Methanogenesis'. Environmental Monitoring and Assessment 42 (1): 57-72.

https://doi.org/10.1007/BF00394042.

Janssen, Stefan, Daniel McDonald, Antonio Gonzalez, Jose A. Navas-Molina, Lingjing Jiang, Zhenjiang Zech Xu, Kevin Winker, et al. 2018. 'Phylogenetic Placement of Exact Amplicon Sequences Improves Associations with Clinical Information'. MSystems 3 (3): e00021-18. https://doi.org/10.1128/mSystems.00021-18.

Kaoutari, Abdessamad El, Fabrice Armougom, Jeffrey I. Gordon, Didier Raoult, and Bernard Henrissat. 2013. 'The Abundance and Variety of Carbohydrate-Active Enzymes in the Human Gut Microbiota'. Nature Reviews Microbiology 11 (7): 497-504. https://doi.org/10.1038/nrmicro3050.

Kaul, Abhishek, Siddhartha Mandal, Ori Davidov, and Shyamal D. Peddada. 2017. 'Analysis of Microbiome Data in the Presence of Excess Zeros'. Frontiers in Microbiology 8. https://doi.org/10.3389/fmicb.2017.02114.

Kelly, Jennifer, Kristian Daly, Andrew W. Moran, Sheila Ryan, David Bravo, and Soraya P. Shirazi-Beechey. 2017. 'Composition and Diversity of Mucosa-Associated Microbiota along the Entire Length of the Pig Gastrointestinal Tract; Dietary Influences'. Environmental Microbiology 19 (4): 1425-38. https://doi.org/10.1111/1462-2920.13619.

Kovatcheva-Datchary, Petia, Anne Nilsson, Rozita Akrami, Ying Shiuan Lee, Filipe De Vadder, Tulika Arora, Anna Hallen, Eric Martens, Inger Björck, and Fredrik Bäckhed. 2015. 'Dietary Fiber-Induced Improvement in Glucose Metabolism Is Associated with Increased Abundance of Prevotella'. Cell Metabolism 22 (6): 971-82. https://doi.org/10.1016/j.cmet.2015.10.001.

Lauber, Christian L., Nicholas Zhou, Jeffrey I. Gordon, Rob Knight, and Noah Fierer. 2010. 'Effect of Storage Conditions on the Assessment of Bacterial Community Structure in Soil and Human-Associated Samples'. FEMS Microbiology Letters 307 (1): 80-86. https://doi.org/10.1111/j.1574-6968.2010.01965.x.

Lennon, J. T., M. E. Muscarella, S. A. Placella, and B. K. Lehmkuhl. 2018. 'How, When, and Where Relic DNA Affects Microbial Diversity'. MBio, June. https://doi.org/10.1128/mBio.00637-18.

Lozupone, C., and R. Knight. 2005. 'UniFrac: A New Phylogenetic Method for Comparing Microbial Communities'. Applied and Environmental Microbiology 71 (12): 8228-35. https://doi.org/10.1128/AEM.71.12.8228-8235.2005.

Marotz, Clarisse, Anukriti Sharma, Greg Humphrey, Neil Gottel, Christopher Daum, Jack A Gilbert, Emiley Eloe-Fadrosh, and Rob Knight. 2019. 'Triplicate PCR Reactions for 16S RRNA Gene Amplicon Sequencing Are Unnecessary'. BioTechniques, May. https://doi.org/10.2144/btn-2018-0192.

McMurdie, Paul J., and Susan Holmes. 2013. 'Phyloseq: An R Package for Reproducible Interactive Analysis and Graphics of Microbiome Census Data'. PLOS ONE 8 (4): e61217. https://doi.org/10.1371/journal.pone.0061217.

Mitchell, Kieren J., Renae C. Pratt, Laura N. Watson, Gillian C. Gibb, Bastien Llamas, Marta Kasper, Janette Edson, et al. 2014. 'Molecular Phylogeny, Biogeography,

Peer) reviewing PDF | (2021:09:65718:2:0:NEW 25 Jan 2022) 
515

516

517

518

519

520

521

522

523

524

525

526

527

528

529

530

531

532

533

534

535

536

537

538

539

540

541

542

543

544

545

546

547

548

549

550

551

552

553

554

555

556

557

558

559

560

and Habitat Preference Evolution of Marsupials'. Molecular Biology and Evolution 31 (9): 2322-30. https://doi.org/10.1093/molbev/msu176.

Neumann, Anthony P., Caroline A. McCormick, and Garret Suen. 2017. 'Fibrobacter Communities in the Gastrointestinal Tracts of Diverse Hindgut-Fermenting Herbivores Are Distinct from Those of the Rumen'. Environmental Microbiology 19 (9): 3768-83. https://doi.org/10.1111/1462-2920.13878.

Pang, Wanyong, Finn Kvist Vogensen, Dennis Sandris Nielsen, and Axel Kornerup Hansen. 2012. 'Faecal and Caecal Microbiota Profiles of Mice Do Not Cluster in the Same Way'. Laboratory Animals 46 (3): 231-36. https://doi.org/10.1258/la.2012.011128.

Quast, Christian, Elmar Pruesse, Pelin Yilmaz, Jan Gerken, Timmy Schweer, Pablo Yarza, Jörg Peplies, and Frank Oliver Glöckner. 2013. 'The SILVA Ribosomal RNA Gene Database Project: Improved Data Processing and Web-Based Tools'. Nucleic Acids Research 41 (Database issue): D590-96. https://doi.org/10.1093/nar/gks1219.

R Core Team. 2020. 'R: A Language and Environment for Statistical Computing'. R Foundation for Statistical Computing. https://www.R-project.org.

Rowland, Ian, Glenn Gibson, Almut Heinken, Karen Scott, Jonathan Swann, Ines Thiele, and Kieran Tuohy. 2018. 'Gut Microbiota Functions: Metabolism of Nutrients and Other Food Components'. European Journal of Nutrition 57 (1): 124. https://doi.org/10.1007/s00394-017-1445-8.

Shiffman, Miriam E., Rochelle M. Soo, Paul G. Dennis, Mark Morrison, Gene W. Tyson, and Philip Hugenholtz. 2017. 'Gene and Genome-Centric Analyses of Koala and Wombat Fecal Microbiomes Point to Metabolic Specialization for Eucalyptus Digestion'. PeerJ 5 (November): e4075. https://doi.org/10.7717/peerj.4075.

Song, Se Jin, Amnon Amir, Jessica L. Metcalf, Katherine R. Amato, Zhenjiang Zech Xu, Greg Humphrey, and Rob Knight. 2016. 'Preservation Methods Differ in Fecal Microbiome Stability, Affecting Suitability for Field Studies'. MSystems 1 (3). https://doi.org/10.1128/mSystems.00021-16.

Stevens, C. Edward, and Ian D. Hume. 1998. 'Contributions of Microbes in Vertebrate Gastrointestinal Tract to Production and Conservation of Nutrients'. Physiological Reviews 78 (2): 393-427. https://doi.org/10.1152/physrev.1998.78.2.393.

Sun, Dong-Lei, Xuan Jiang, Qinglong L. Wu, and Ning-Yi Zhou. 2013. 'Intragenomic Heterogeneity of 16S RRNA Genes Causes Overestimation of Prokaryotic Diversity'. Applied and Environmental Microbiology 79 (19): 5962-69. https://doi.org/10.1128/AEM.01282-13.

Thompson, Luke R., Jon G. Sanders, Daniel McDonald, Amnon Amir, Joshua Ladau, Kenneth J. Locey, Robert J. Prill, Anupriya Tripathi, Sean M. Gibbons, and Gail Ackermann. 2017. 'A Communal Catalogue Reveals Earth's Multiscale Microbial Diversity'. Nature 551 (7681): 457-63.

Ubeda, Carles, Ana Djukovic, and Sandrine Isaac. 2017. 'Roles of the Intestinal Microbiota in Pathogen Protection'. Clinical \& Translational Immunology 6 (2): e128. https://doi.org/10.1038/cti.2017.2.

Vasapolli, Riccardo, Kerstin Schütte, Christian Schulz, Marius Vital, Dirk Schomburg, Dietmar H. Pieper, Ramiro Vilchez-Vargas, and Peter Malfertheiner. 2019. 'Analysis of Transcriptionally Active Bacteria Throughout the Gastrointestinal 
$561 \quad$ Tract of Healthy Individuals'. Gastroenterology 157 (4): 1081-1092.e3. $562 \quad$ https://doi.org/10.1053/j.gastro.2019.05.068.

563 Weiss, Sesilje, David Taggart, lan Smith, Kristofer M. Helgen, and Raphael Eisenhofer.

564

565

566

567

568

569

570

571

572 2021. 'Host Reproductive Cycle Influences the Pouch Microbiota of Wild Southern Hairy-Nosed Wombats (Lasiorhinus Latifrons)'. Animal Microbiome 3 (1): 13. https://doi.org/10.1186/s42523-021-00074-8.

Wells, Rod. 1989. 'Vombatidae'. In Fauna of Australia Volume 1B Mammalia, 755-68. Australian Government Publishing Service, Canberra, Australian Capital Territory, Australia.

Wickham, Hadley. 2016. Ggplot2: Elegant Graphics for Data Analysis. Springer-Verlag New York. https://ggplot2.tidyverse.org.

\section{Acknowledgements}

574 The authors would like to thank Alejandro Correa Lozano for his help collecting and analysing 576 the BNW samples. 
Figure 1

Illustration of wombat gut tracts and samples collected

Drawn illustrations of Vombatus ursinus (top) and Lasiorhinus latifrons (bottom)

gastrointestinal tracts based on Barboza and Hume 1992a. Stars represent locations where duplicate samples were collected for the study. BNW = Bare-nosed wombat, SHNW = Southern hairy-nosed wombat.

Vombatus ursinus (BNW)

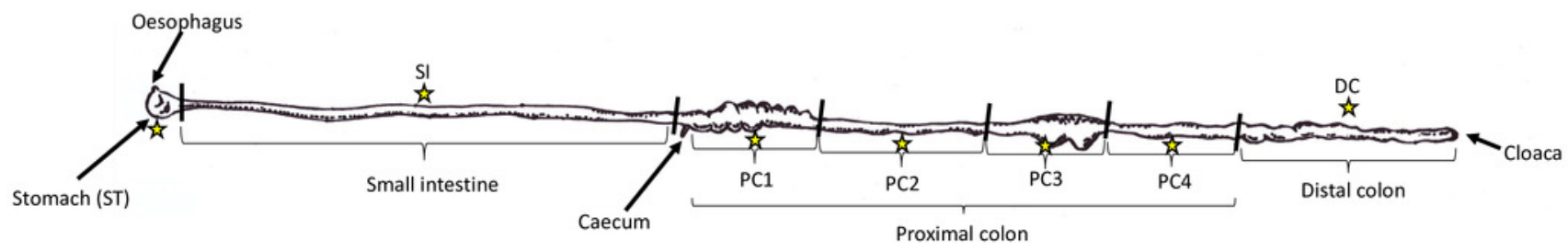

Lasiorhinus latifrons (SHNW)

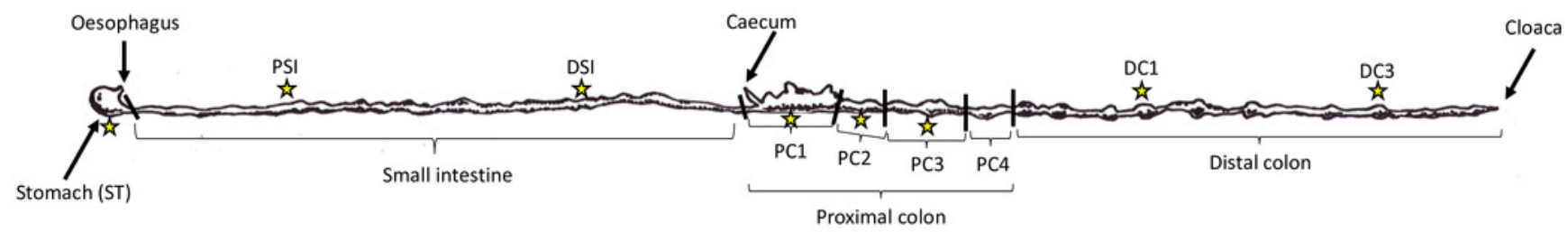


Figure 2

Taxonomic composition of the different sample types at the family level

Only the top 20 most abundant families are displayed for clarity (these families account for $81.17 \%$ of reads) The 'Other' bin (grey) contains the other families. Replicate samples were merged per sample site. A) bare-nosed wombat. B) southern hairy-nosed wombat. The widths of the bars are scaled to the length of the Gl region.

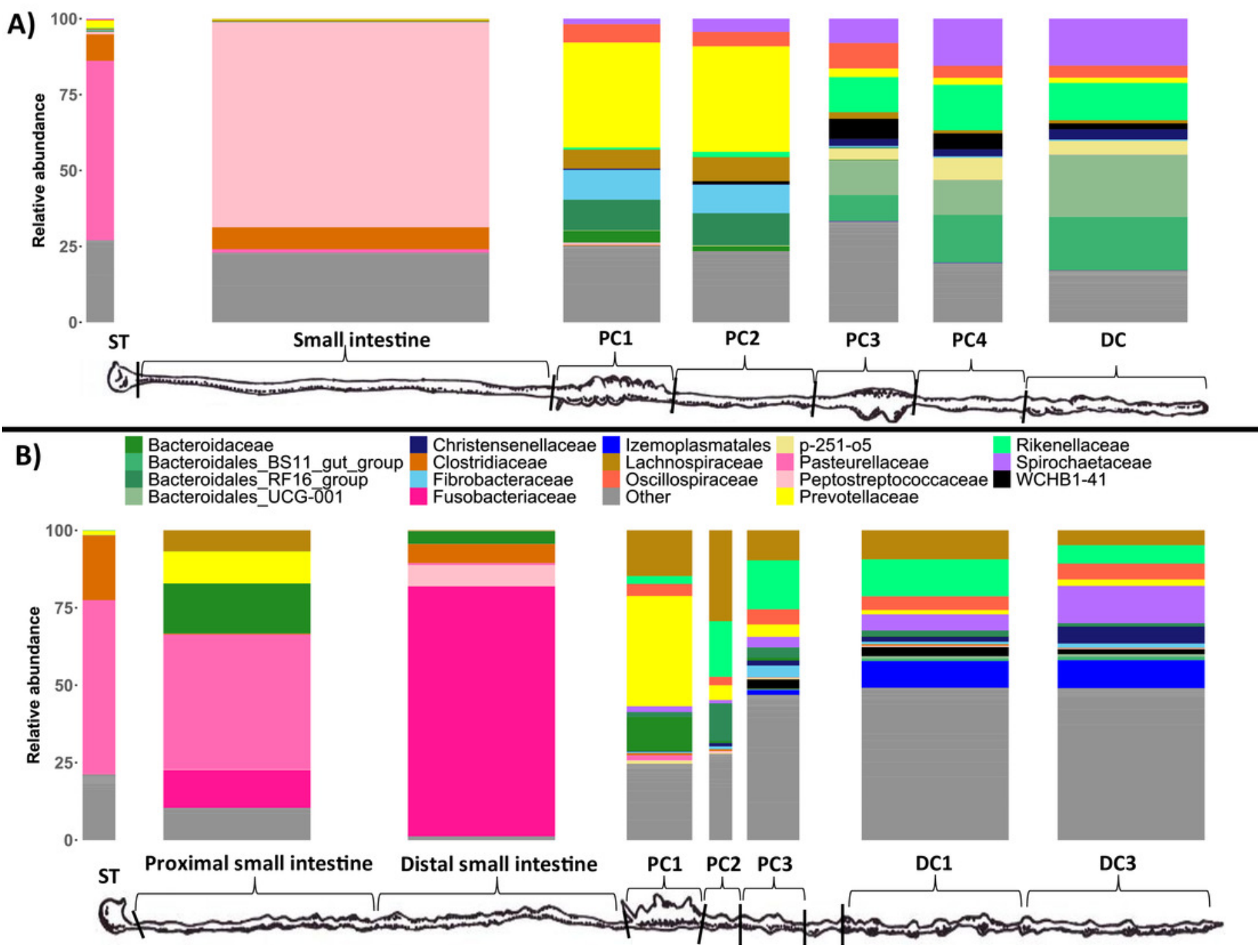




\section{Figure 3}

Microbial diversity (ASV richness) of samples collected throughout the wombat digestive tract, ordered from start to end.

$\mathrm{ST}=$ stomach, $\mathrm{SI}=$ small intestine, $\mathrm{PSI}=$ proximal small intestine, $\mathrm{DSI}=$ distal small intestine, $\mathrm{PC}=$ proximal colon, $\mathrm{DC}=$ distal colon. Two technical replicates were collected and processed for each site (joined by lines). A) bare-nosed wombat. B) southern hairy-nosed wombat. Digestive tract drawings were adapted from Barboza and Hume 1992a.

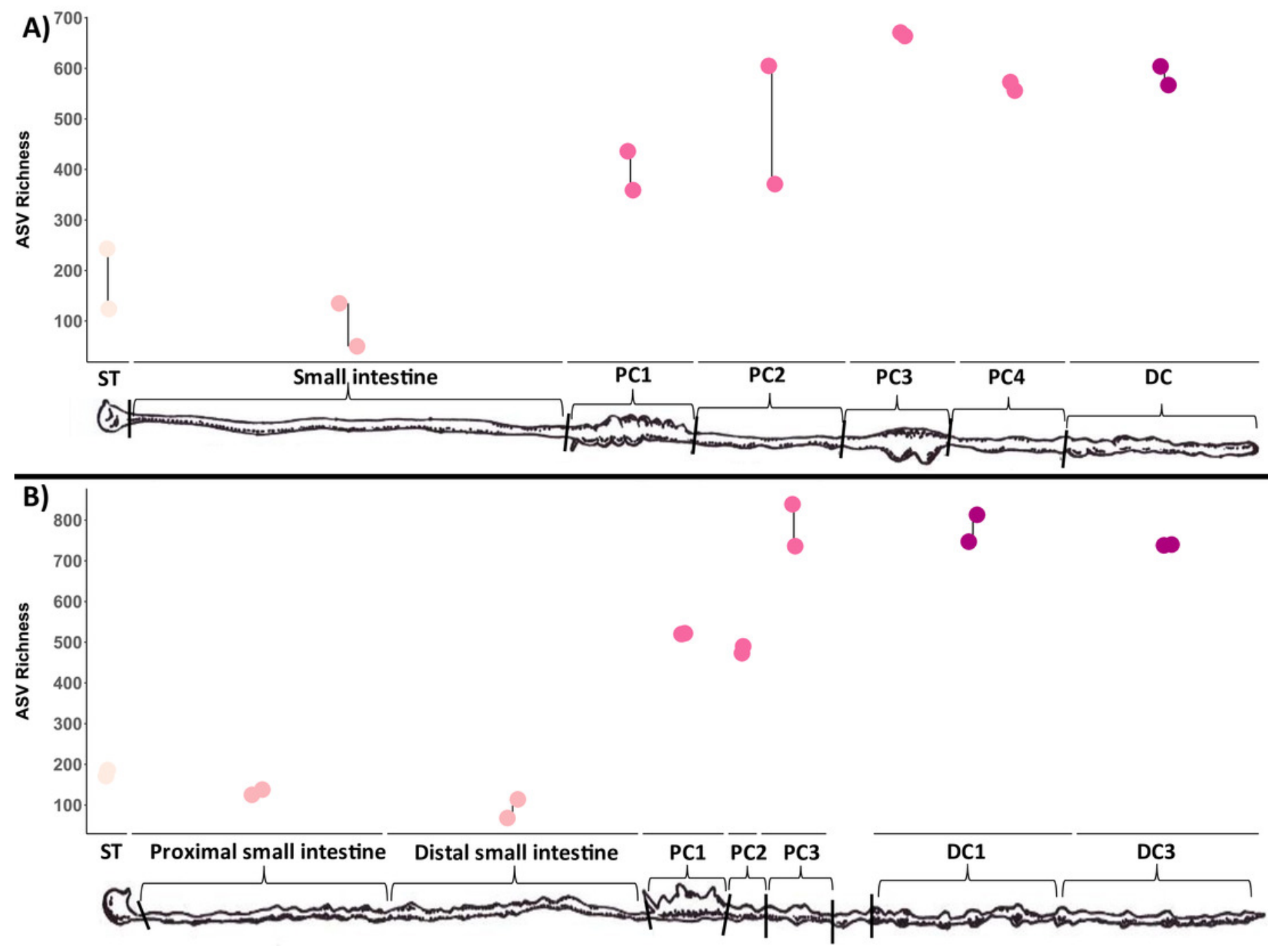


Figure 4

Microbial community structure among sites along the wombat GI tract.

A) PCoA of unweighted UniFrac distances and B) PCoA of weighted UniFrac distances.

Samples are coloured by sample type, and shaped by host species. Numbers indicate order in which samples occur in the digestive tract. Lines connect samples from the same sample type. 

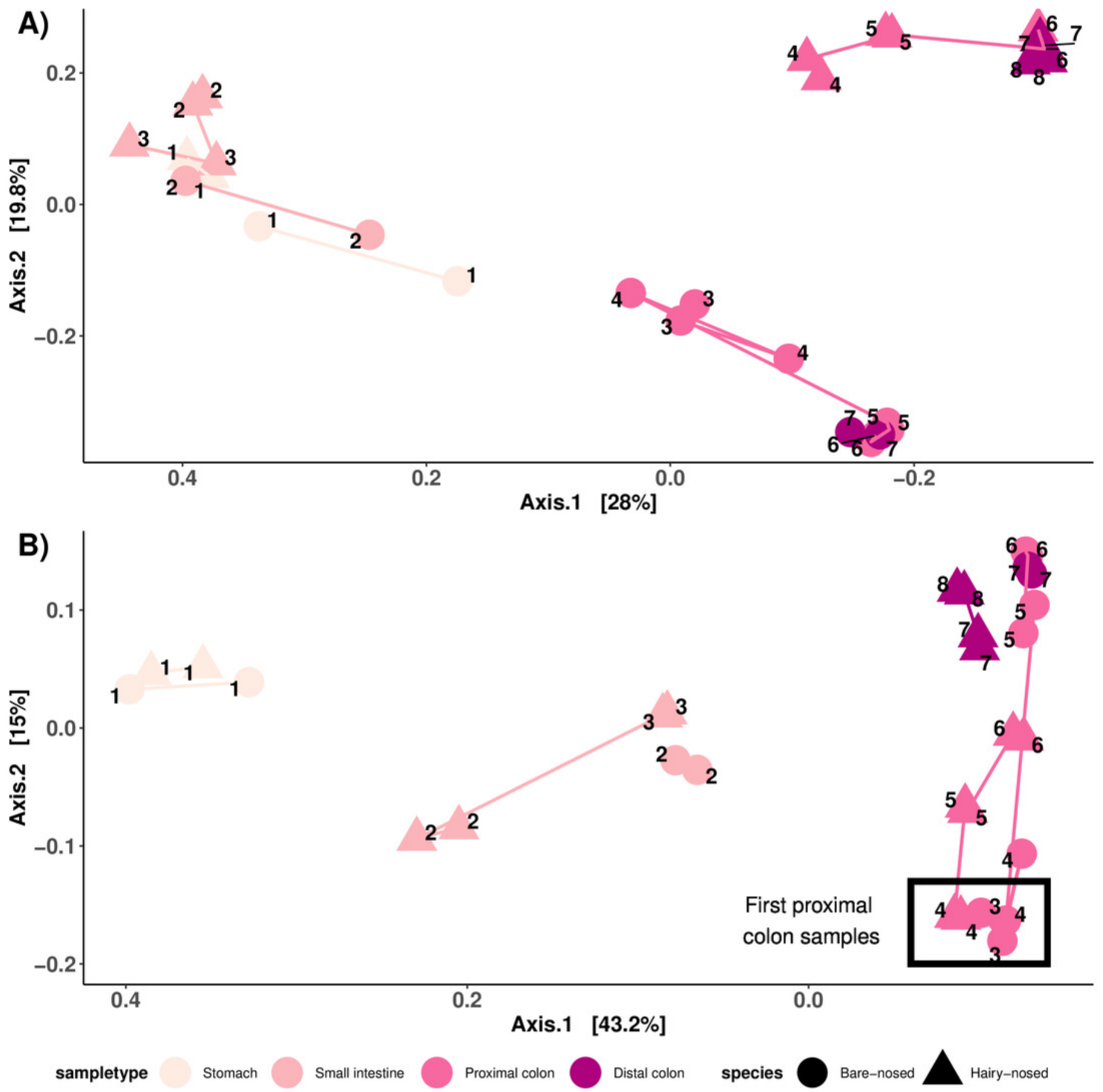


\section{Figure 5}

Heat map of the microbial genera that were found to be differentially abundant between proximal and distal colon sites.

The abundance of genera that were found to be differentially abundant ( 0.7 threshold) in the ANCOM-II analysis are displayed. Black indicates 0 assigned reads. Non-genus assignments are prefixed with the lowest level of taxonomy that could be assigned (e.g. $f_{-}=$family). Taxonomy string are prefixed with 'Phylum -- '. 


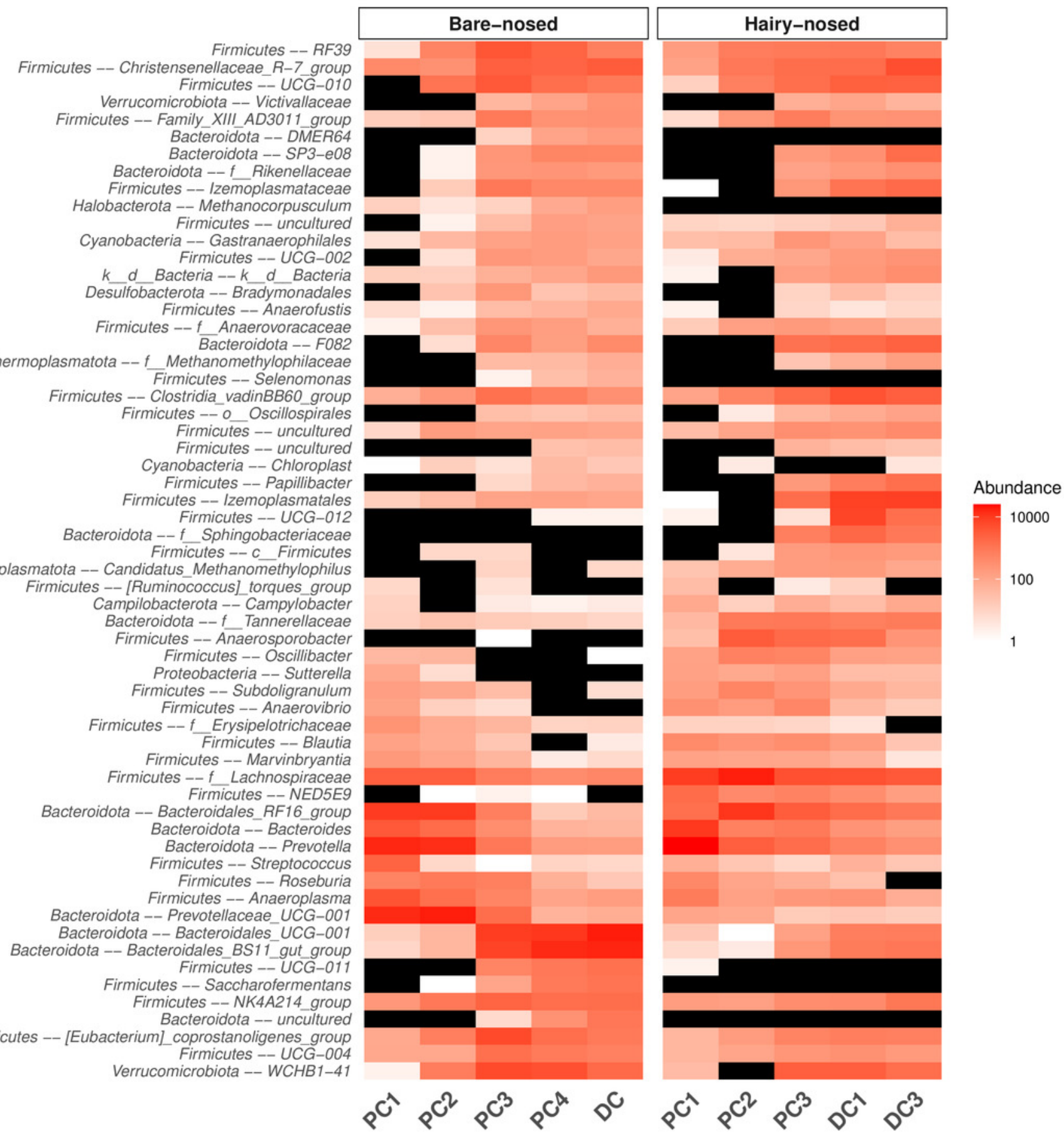




\section{Figure 6}

Euler diagram of amplicon sequence variants (ASVs) shared between proximal and distal colon sites for both wombat species.

Euler diagram of amplicon sequence variants (ASVs) shared between proximal colon 1 (PC1) and last distal colon site (DC) for A) bare-nosed wombat (BNW) and B) southern hairy-nosed wombat (SHNW). Percentages represent the proportion of ASVs specific to a given area. 
A) Bare-nosed wombat

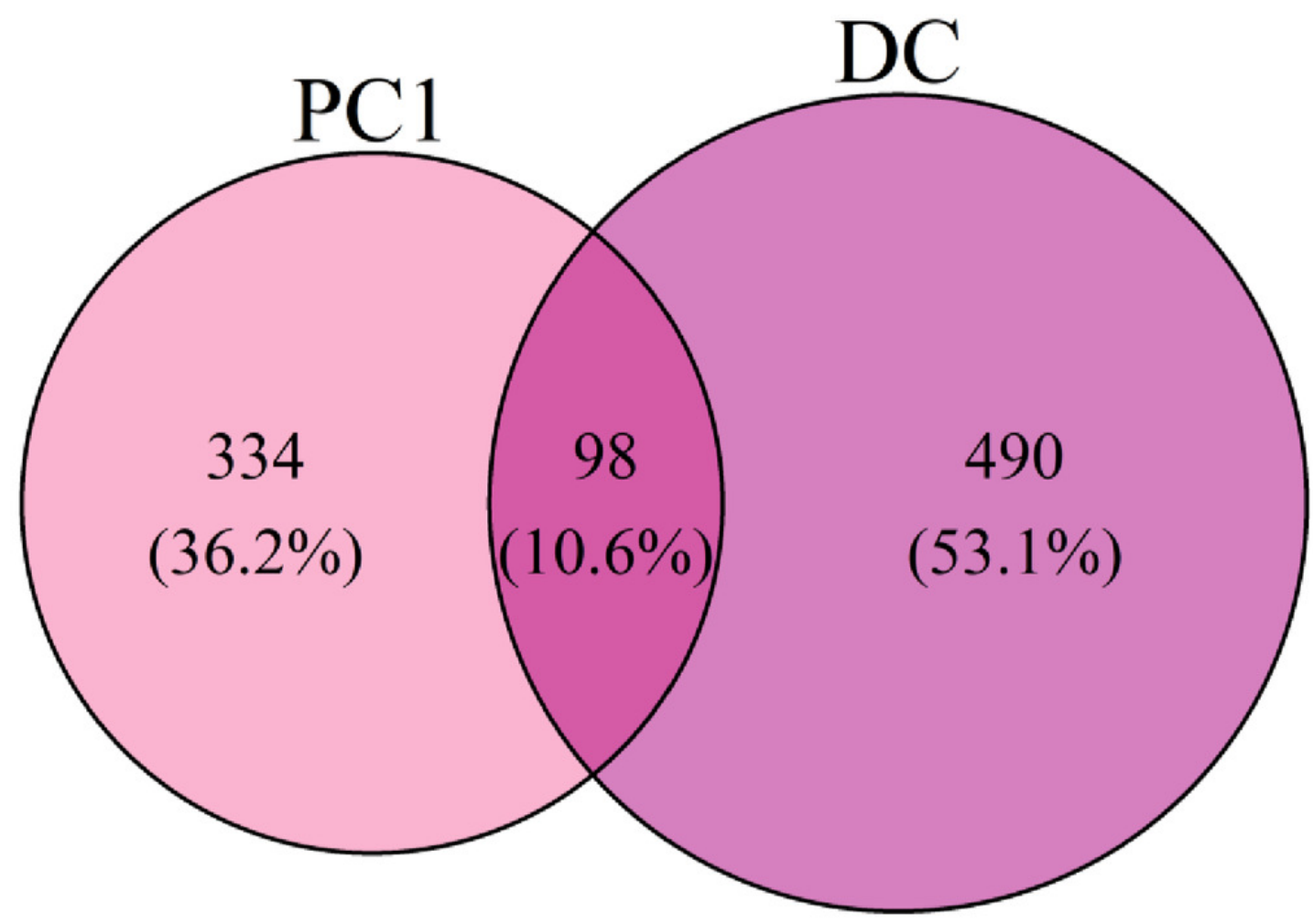

B) Southern hairy-nosed wombat

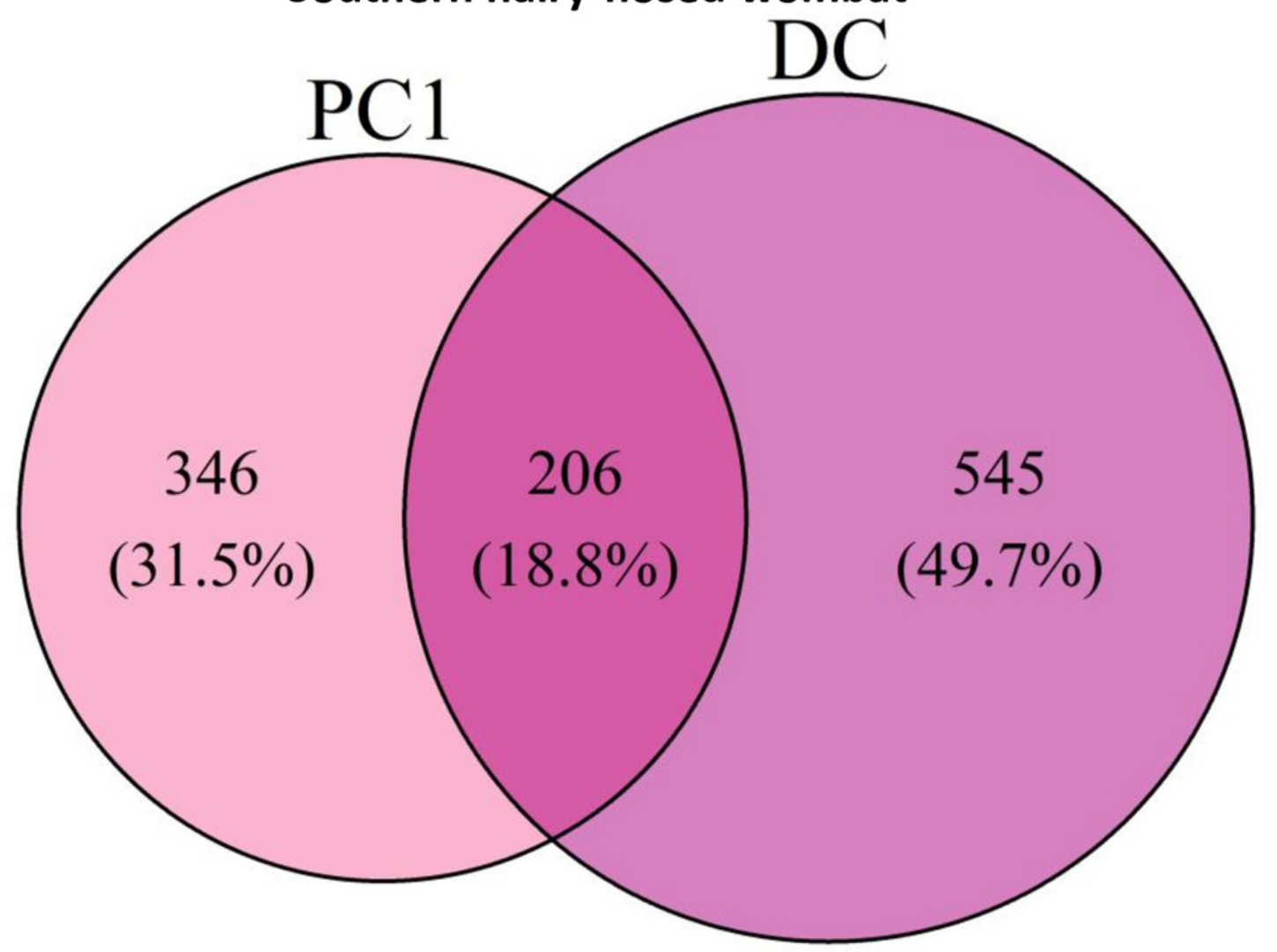

